\title{
La rétention des feuilles mortes dans un cours d'eau de montagne, au Maroc : variations saisonnières
}
A Maamri 1
H. Cherguil
E. Pattee 2

Mots clés : débit, rétention, saison, dilacérateurs.

Dans le but d'examiner l'effet du débit sur la rétention, la méthode de marquage, lâcher et recapture de feuilles marquées (Salix pedicellata et Nerium oleander à l'état frais et à l'état sec) a été appliquée dans deux stations de l'Oued Zeghzel (la Branche Sud-Ouest et le Bas Zeghzel) aux quatre saisons de l'année 1991-1992.

Les feuilles lâchées durant les saisons à fort débit (automne et hiver) étaient moins retenues que celles lâchées durant les saisons à débit faible (printemps et été). Dans le Bas Zeghzel en particulier, le pourcentage des feuilles retenues augmentait visiblement lorsque le débit diminuait.

La rétention en fonction de la distance répondait à un modèle exponentiel négatif et à un modèle linéaire (dispersion aléatoire des feuilles retenues). Mais dans l'ensemble, le premier décrivait mieux les conditions de plus faible débit, le second celles de plus fort débit.

Les petits barrages de débris de bois, la végétation riveraine et les branchages étaient les structures de rétention les plus efficaces. Leur efficacité augmentait lorsque le débit diminuait. Quelle que soit la saison d'étude, elles hébergeaient une faune variée et très riche en dilacérateurs dans le secteur amont du Zeghzel. Elles étaient plus rares et moins peuplées dans le secteur aval.

\section{Seasonal variations in the retention of dead leaves in a Moroccan mountain stream}

Keywords : discharge, retention, season, shredders.

In order to investigate the influence of discharge on leaf retention, batches of fresh and dried leaves of two species (Salix pedicellata and Nerium oleander) were stained and released in four consecutive seasons, from autumn 1991 to summer 1992. Release took place in two $50 \mathrm{~m}$-long reaches of Oued Zeghzel, the Southwestern Branch (a headwater stream overhung by dense vegetation) and the Lower Zeghzel (with higher discharge and sparse riparian vegetation). Each reach was closed downstream by a net.

Retention versus distance conformed both with a negative exponential model and with a linear (random retention) model. It was higher and altogether better described by an exponential model in seasons with low discharge than in seasons with high discharge. All leaves were retained within $5 \mathrm{~m}$ (summer) to $26 \mathrm{~m}$ (winter) in the Southwestern Branch. Leaves were always caught in the downstream net of the Lower Zeghzel and extrapolation of the curves showed that they should have drifted over several hundred metres, but the percentage of leaves retained within the reach increased while discharge decreased.

The most retentive structures were small debris dams, submerged riparian vegetation and branches. Their retentive efficiency increased versus decreasing discharge. In the Southwestern Branch whatever the season, these structures were numerous and inhabited by a diverse macroinvertebrate fauna, among which shredders strongly dominated both in numbers and in biomass. Retentive structures were scarce and less inhabited in the Lower Zeghzel.

1. Laboratoire d'Hydrobiologie et Ecologie Générale, Faculté des Sciences, Université Mohamed Premier, Oujda, Maroc.

2. Laboratoire de Biologie Animale et Ecologie, Université Claude Bernard Lyon-I, 43 bd du 11 novembre, 69622 Villeurbanne Cedex, France (URA CNRS 1451 : Ecologie des Eaux Douces et des Grands Fleuves). 


\section{Introduction}

L'accumulation de la matière végétale d'origine allochtone constitue un pool d'énergie et de nutriments pour les différentes biocénoses qui occupent les écosystèmes aquatiques. Cette accumulation dépend aussi bien de l'apport de la matière végétale terrestre et de sa rétention que de sa vitesse de décomposition.

Si la décomposition de cette matière végétale a fait l'objet de nombreuses publications (Fisher \& Likens 1973, Post \& Cruz 1977, Cruz \& Post 1977, Cummins et al. 1989, Webster \& Benfield 1986, Pattee et al. 1986, Chergui \& Pattee 1988, 1991, Boulton \& Boon 1991, Oertli 1991), les études sur sa rétention restent limitées, bien qu'elles aient préoccupé quelques auteurs ces dernières années (Speaker et al. 1984, Speaker et al. 1988, Petersen \& Petersen 1991, Prochazka et al. 1991, Snaddon et al. 1992).

La quantité de matériel végétal particulaire pouvant être retenue dépend principalement des caractéristiques hydrologiques du ruisseau et de la structure de son lit (Speaker et al. 1984, Prochazka et al. 1991, Petersen \& Petersen 1991). Le débit, en particulier, doit jouer dans ce processus un rôle déterminant en affectant la proportion d'eau libre et l'irrégularité du lit, déterminant de ce fait la capacité de rétention du chenal tout entier (Snaddon et al. 1992).

Ce processus de rétention est aussi susceptible d'influencer la distribution des invertébrés. Winterbourn et al. (1981) ont suggéré qu'une grande densité de dilacérateurs reflète l'efficacité de rétention du système aquatique, alors que leur absence témoigne d'une faible rétention. Cummins et al. (1989) ont confirmé cette corrélation.

Récemment, en s'interrogeant sur la répartition amont-aval de la faune invertébrée aquatique, Chergui et al. (1993) ont signalé la différence de rétention également apparue dans deux secteurs d'une petite rivière marocaine, l'Oued Zeghzel, lors d'une expérience ponctuelle, réalisée en 1991.

Le but de cet article est de suivre le processus de rétention des particules grossières de matière organique pendant les quatre saisons de l'année afin de mieux caractériser l'influence des variations du débit sur ce processus et éventuellement sur la distribution des invertébrés dilacérateurs. Plus précisément, un abaissement du débit peut-il augmenter le potentiel de rétention d'un système peu rétentif et, par suite, favoriser le développement temporaire des dilacérateurs .?

\section{Matériel et méthodes}

\subsection{Stations d'étude}

L'oued Zeghzel est un affluent du cours inférieur de la Moulouya, au Nord-Est du Maroc. La carte des sites d'étude dans la Branche Sud-Ouest (Haut Zeghzel, ruisseau de montagne) et dans le Bas Zeghzel est donnée par Chergui \& Pattee (1991). Les caractéristiques de ces stations aux quatre campagnes annuelles (10.11.1991, 15.01.1992, 15.04.1992, 15.07.1992) sont décrites dans le tableau I. Le débit $\mathrm{D}$ a été évalué, à partir de la vitesse superfícielle $\mathrm{V}$ et de la section mouillée $S$ dans les zones les plus étroites, par l'équation $\mathrm{D}=\mathrm{V} \times \mathrm{S}$. Le rayon hydraulique a été calculé par la formule

$$
\mathbf{R}=\mathbf{L} \times \mathbf{P} /(2 \mathrm{P}+\mathrm{L}),
$$

dans laquelle $L$ représente la largeur et $P$ la profondeur moyenne. Le lit de la Branche Sud-Ouest est constitué de galets, graviers et sable avec des gros blocs et de nombreux barrages de détritus végétaux. Il est bordé de saule (Salix pedicellata Desf.), de laurier rose (Nerium oleander L.) et de roseaux (Arundo donax L.). Le Bas Zeghzel coule dans des gorges. Le fond comprend des galets, de la vase et du sable, avec de gros blocs. La végétation des rives, constituée surtout de laurier rose, est rare et espacée. Au niveau de chacun de ces secteurs, un tronçon de $50 \mathrm{~m}$ de long a été choisi et barré à l'aval par un filet de mailles $1 \mathrm{~mm}$.

\subsection{Méthodes}

La méthode est celle utilisée par Chergui et al. (1993). Le lit de chaque tronçon a été divisé selon ses caractéristiques, son substrat minéral et toute autre structure de rétention pouvant être identifiée. Les structures répertoriées sont les blocs submergés ou partiellement émergés, les cailloux et les galets, le gravier au fond de l'eau, la végétation riveraine submergée, les branches, les petits barrages de débris de bois, et les bassins stagnants. Les branches sont généralement des structures ramifiées portant des tiges et des feuilles à travers lesquelles l'eau coule librement. Les petits barrages de débris de bois sont des structures hétérogènes incluant des tiges, des feuilles, des branches et des cailloux, qui interrompent le courant et créent des petites flaques d'eau à leur amont immédiat. Le reste du substrat est 
Tableau I. Caractères morphodynamiques des deux stations lors des quatre campagnes d'étude. RH = rayon hydraulique (définition dans le texte).

Chaque valeur correspond à la moyenne de plusieurs mesures réalisées durant une même journée sur des transects différents des deux stations.

Table I. Morphodynamic characteristics of the two reaches during the four sampling periods.

RH $=$ hydraulic radius (definition in the text).

Each figure is the mean of several estimations performed in different transects of both stations on the same day.

\begin{tabular}{|c|c|c|c|c|c|c|c|c|c|c|}
\hline & \multicolumn{5}{|c|}{ Branche Sud-Ouest } & \multicolumn{5}{|c|}{ Bas Zeghzel } \\
\hline & $\begin{array}{c}\text { profondeur } \\
\mathrm{cm}\end{array}$ & $\begin{array}{c}\text { largeur } \\
\mathrm{cm}\end{array}$ & $\begin{array}{l}\text { débit } \\
1 \mathrm{~s}^{-1}\end{array}$ & $\begin{array}{r}\text { vitesse } \\
\mathrm{m} \mathrm{s}^{-1}\end{array}$ & $\mathbf{R H}$ & $\begin{array}{c}\text { profondeur } \\
\mathrm{cm}\end{array}$ & $\begin{array}{c}\text { largeur } \\
\mathrm{cm}\end{array}$ & $\begin{array}{c}\text { débit } \\
1 \mathrm{~s}^{-1} \\
\end{array}$ & $\begin{array}{c}\text { vitesse } \\
\mathrm{m} \mathrm{s}^{-1}\end{array}$ & $\mathrm{RH}$ \\
\hline Automne & 14 & 201 & 86 & 0,47 & 12 & 24 & 425 & 280 & 0,16 & 18 \\
\hline Hiver & 15 & 206 & 98 & 0,74 & 13 & 24 & 400 & 319 & 0,28 & 21 \\
\hline Printemps & 12 & 175 & 35 & 0,38 & 10 & 17 & 298 & 116 & 0,23 & 15 \\
\hline Eté & 11 & 146 & 24 & 0,25 & 9 & 17 & 272 & 90 & 0,18 & 15 \\
\hline
\end{tabular}

généralement de la vase et du sable. Les structures de rétention ont été cartographiées et le pourcentage de surface occupé par chacune dans chaque station a été calculé. L'efficacité de rétention est évaluée comme le rapport du pourcentage des feuilles retenues par une structure sur la surface relative occupée par cette même structure dans chaque station (Prochazka et al. 1991). Des feuilles de Salix pedicellata et de Nerium oleander, les unes fraîches et les autres préalablement séchées pendant 48 heures à $40^{\circ} \mathrm{C}$, ont été marquées au colorant indélébile. Leur rétention a été évaluée aux quatre saisons de l'année : la position de 200 feuilles de chaque catégorie a été relevée 48 heures après leur déversement en amont de chaque secteur. En effet, Chergui et al. (1993) avaient montré que la localisation des feuilles dans quelques structures après $3 \mathrm{~h}$ n'était que provisoire.

Les données sont confrontées au modèle exponentiel négatif de rétention, d'équation (Young et al. 1978) :

$$
N_{d}=N_{0} e^{-k d}
$$

où $\mathrm{d}=$ la distance parcourue par les feuilles, en mètres,

$\mathbf{N}_{\mathbf{d}}=$ le nombre de feuilles retenues à la distance $d$,

$\mathrm{N}_{0}=$ le nombre de feuilles introduites en amont,

$\mathbf{k}=$ le coefficient de rétention par mètre.
Ce modèle implique que chaque unité successive de longueur du ruisseau retient une fraction identique des feuilles en dérive qui lui parviennent.

La correspondance avec un modèle linéaire

a également été testée.

$$
N_{d}=N_{0^{-}} \mathbf{a d}
$$

La macrofaune aquatique a été récoltée à l'aide d'un filet Surber permettant d'échantillonner une surface de $0,1 \mathrm{~m}^{2}$ dans chacune des structures de rétention. Elle a été répartie entre dilacérateurs et autres invertébrés d'après Merrit \& Cummins (1978), Tachet et al. (1980) et Cummins \& Wilzbach (1985). Le poids sec de la faune a été évalué après passage à $110^{\circ} \mathrm{C}$ pendant 24 heures. Vu la taille minuscule de certains d'entre eux, les gastéropodes ont été pesés avec leur coquille.

\section{Résultats}

Aux quatre campagnes, le débit ainsi que la largeur moyenne et la profondeur qui y sont étroitement liés, montrent des valeurs plus importantes dans le Bas Zeghzel que dans le secteur amont (Tableau I). Le rayon hydraulique, indice d'irrégularité du chenal, est légèrement plus élevé en aval qu'en amont. En fonction du débit, on peut distinguer grossièrement deux groupes de campagnes : celles caractérisées par un débit important en automne et en hiver, et celles à faible débit au printemps et en été.

Dans la Branche Sud-Ouest (Fig. 1), un pourcentage moyen de $98 \%$ de feuilles (à la fois celles de 
Salix et de Nerium) a été retrouvé sur le fond, donc manifestement retenu, durant la période à débit important (automne et hiver). Durant la période printemps-été caractérisée par un faible débit, le pourcentage des feuilles retrouvées est de $100 \%$. A aucune saison des feuilles n'ont été retrouvées dans le filet. Dans ce secteur, la distance parcourue par les feuilles tend à augmenter par fort débit (Fig. 1), les coefficients de rétention étant alors plus faibles (Tableau 2). Quelle que soit la saison d'étude, les courbes exprimant le nombre de feuilles retenues par

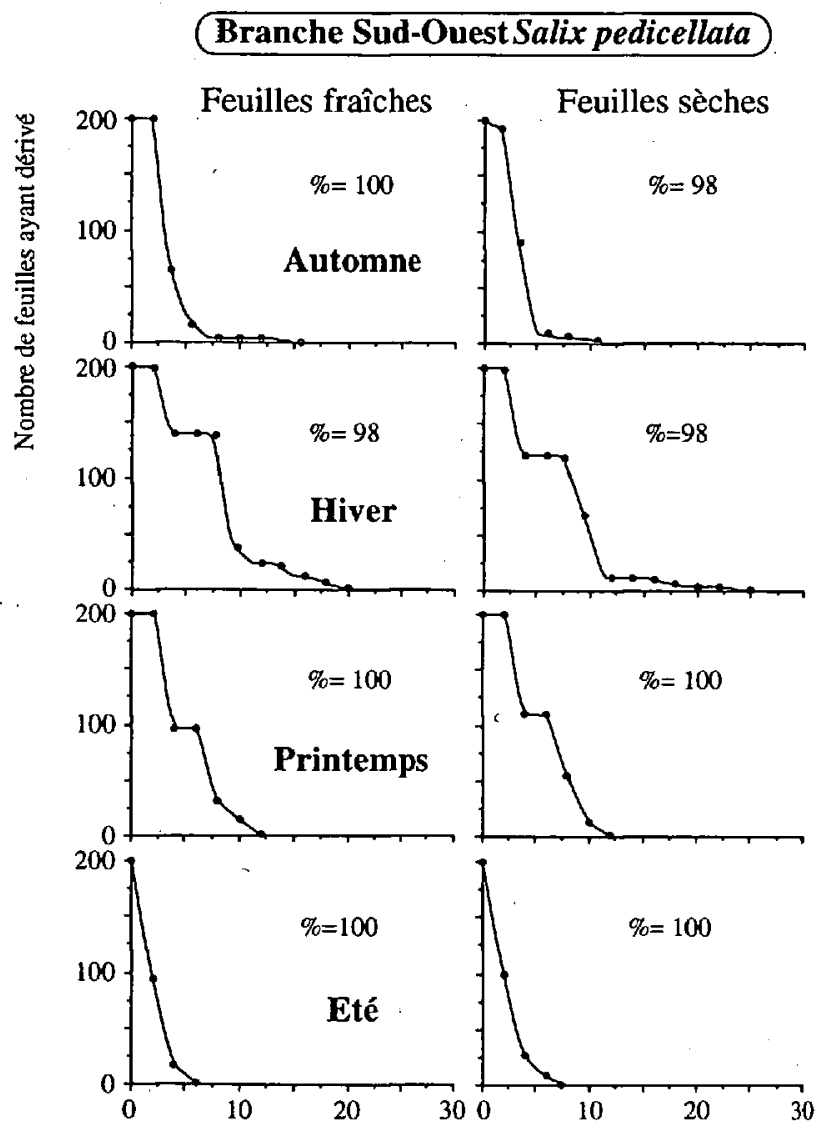

rapport à la distance parcourue sont compatibles avec le modèle exponentiel négatif $(r>0,70)$ et, sauf au printemps (bien que re $\geqslant 0,87$ ) et dans un cas en été ( $\mathrm{re}=\mathrm{rl}=0,96)$, ce modèle décrit mieux la rétention que le simple modèle linéaire. Dans le détail, les tracés de l'hiver, du printemps et moins nettement ceux de l'automne, marquent deux paliers, l'un à $2 \mathrm{~m}$, l'autre entre 4 et 6 ou 4 et $8 \mathrm{~m}$ du départ.

Dans le Baz Zeghzel, une bonne moitié, voire les trois quarts des feuilles atteignent le filet à la limite

\section{Branche Sud-Ouest Nerium oleander}

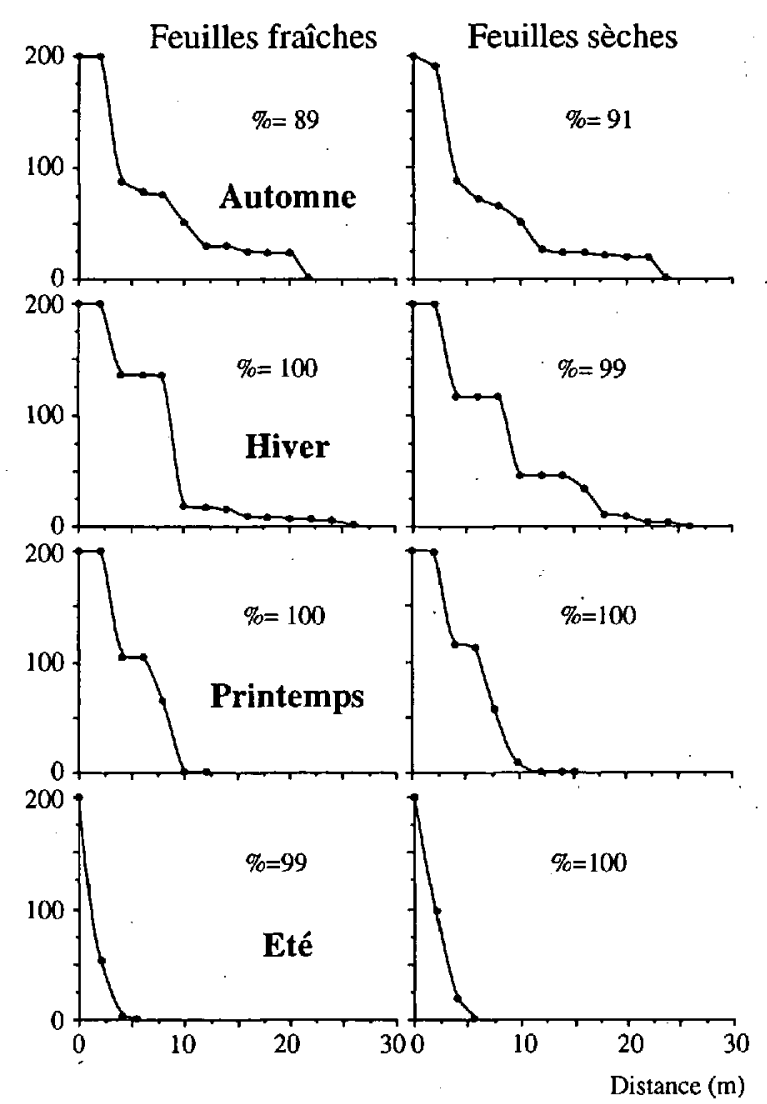

Fig. 1. Nombre de feuilles marquées qui ont dérivé au-dessus de chaque transect du secteur de $50 \mathrm{~m}$ de la Branche Sud-Ouest au cours des $48 \mathrm{~h}$ suivant leur lâcher, calculé d'après les nombres de feuilles retrouvées en aval. Les résultats des quatre campagnes d'étude de l'année 1991/92 sont représentés. Des lots de 200 feuilles de chaque catégorie ( 800 feuilles en tout) ont été lâchés simultanément en amont du secteur.

$\%$ = celui des feuilles récupérées dans le secteur. Aucune feuille n'est arrivée en aval du secteur.

Fig. 1. Number of marked leaves in transport within 48 hours versus distance travelled from the release point in the 50 -m reach of the Southwestern Branch of Oued Zeghzel at each sampling period of the year 1991/92. Batches of 200 leaves of each category ( 800 leaves altogether) were released together at the upstream end of the reach.

$\%=$ percentage of leaves recovered in the reach. No leaves drifted as far as the downstream net. 
Tableau 2. Rétention en 48 heures de feuilles de Salix et de Nerium fraîches et sèches dans les deux stations aux quatre saisons. $\mathrm{SF}=$ feuilles fraîches de Saule

SS = feuilles sèches de Saule

LF = feuilles fraîches de Laurier

LS $=$ feuilles sèches de Laurier

$\max =$ distance maximale effectivement parcourue par les feuilles

$\mathbf{k}=$ coefficient exponentiel de rétention par mètre de longueur du cours d'eau

re $=$ coefficient de corrélation du modèle exponentiel de rétention

$\mathrm{rl}=$ coefficient de corrélation du modèle linéaire de rétention

$\mathrm{D}$ moy = distance moyenne parcourue par les feuilles $(=1 / \mathrm{k})$

$\mathrm{D}$ tot $=$ distance théorique totale nécessaire à la rétention de $99 \%$ des feuilles $(=\ln 100 / \mathrm{k})$

Les distances sont en mètres.

Table 2 : Retention of Salix and Nerium fresh and dry leaves within 48 hours in the two stations during the four seasons.

Branche SO = S.W. Branch

Bas Zeghzel = Lower Zeghzel

$\mathrm{SF}=$ fresh Salix leaves

SS $=$ dry Salix leaves

LF $=$ fresh Nerium leaves

LS = dry Nerium leaves

$\max =$ maximum distance actually drifted by the leaves

$k=$ instantaneous rates of retention per metre along the stream

re $=$ correlation coefficient of the exponential retention model

$r l=$ correlation coefficient of the linear retention model

$\mathrm{D}$ moy $=$ mean distance travelled by the leaves $(=1 / \mathrm{k})$

$\mathrm{D}$ tot $=$ distance within which $99 \%$ of the leaves were retained $(=\ln 100 / \mathrm{k})$.

Distances are in metres.

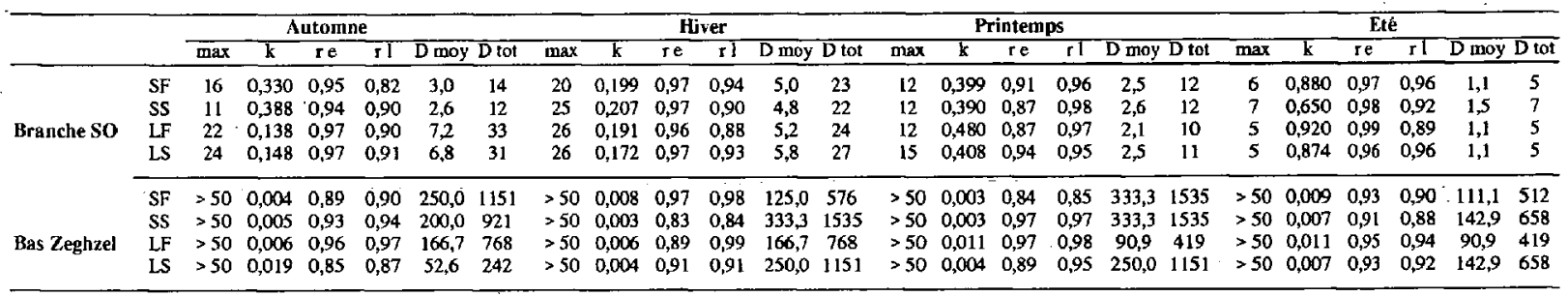

aval du secteur (Fig. 2). Nous constatons que le pourcentage des feuilles retenues varie nettement avec le débit, quelle que soit l'essence végétale. Ce pourcentage moyen est de l'ordre de $24 \%$ en automne et de $22 \%$ en hiver, lorsque le débit est important (280 $1 . \mathrm{s}^{-1}$ et $3191 . \mathrm{s}^{-1}$ respectivement (Tableau 1). Lorsque le débit devient faible durant la période printanière et estivale (116 1.s $\mathrm{s}^{-1}$ et $901 . \mathrm{s}^{-1}$ respectivement), le pourcentage moyen de feuilles retenues croît nettement : $33 \%$ au printemps et $42 \%$ en été. Les coefficients de corrélation avec le modèle exponentiel sont tous supérieurs, et souvent de beaucoup, à 0,80 (Tableau 2) mais sauf en été, lors du plus faible débit, le modèle linéaire décrit mieux la rétention. En effet les courbes ont un aspect plus étalé que celles de l'amont, avec des distances extrapolées de fixation de $99 \%$ des feuilles ( $D$ tot) atteignant plusieurs centaines de mètres, voire même dépassant le kilomètre.

Quoique la plupart des structures de rétention soient présentes dans les deux secteurs d'étude, leur efficacité (évaluée selon sa définition, en pourcentage et non en valeur absolue, Fig. 3) diffère considérablement d'un secteur à l'autre et aussi dans un même secteur en fonction de la saison d'étude et donc du débit. Ainsi, les petits barrages de débris de bois, présents d'ailleurs uniquement dans le secteur amont, se révèlent comme la structure de rétention la plus efficace durant les quatre saisons. Mais cette efficacité augmente nettement lorsque l'on passe de l'automne à l'été et que le débit diminue. La même constatation est faite, dans les deux secteurs, pour la végétation riveraine et les branchages, 
Bas Zeghzel Salix pedicellata

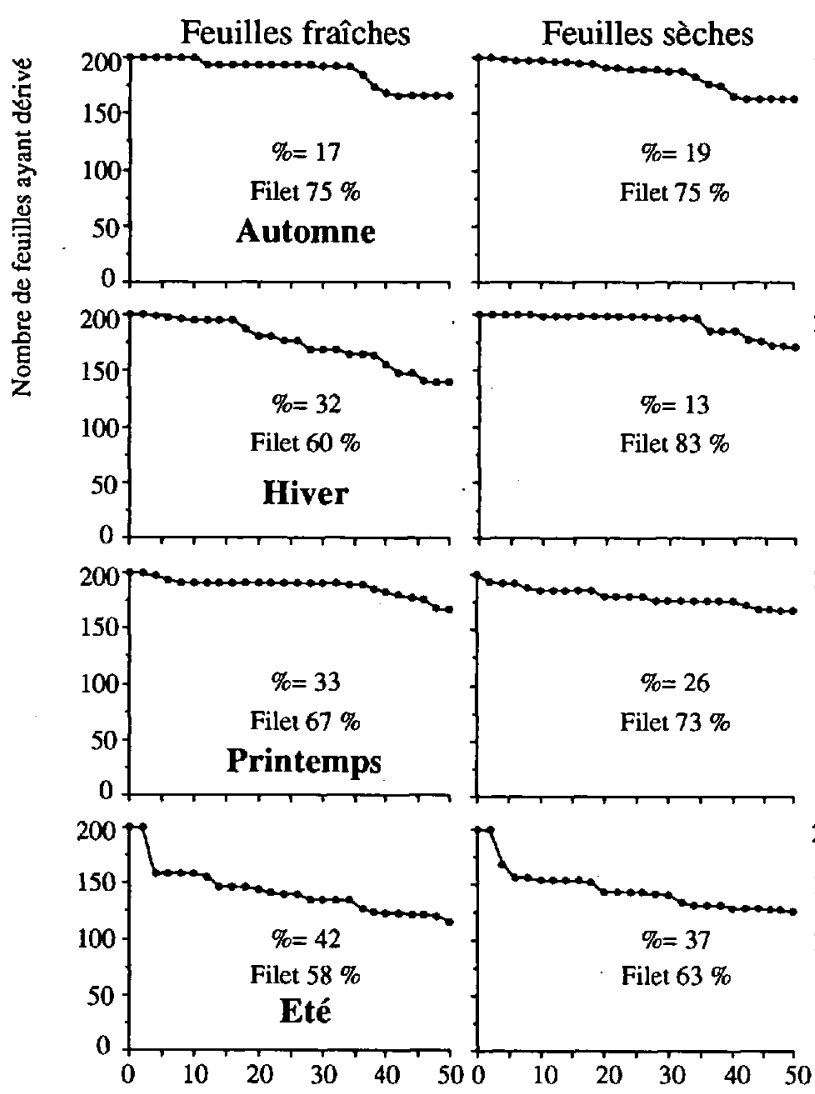

Bas Zeghzel Nerium oleander
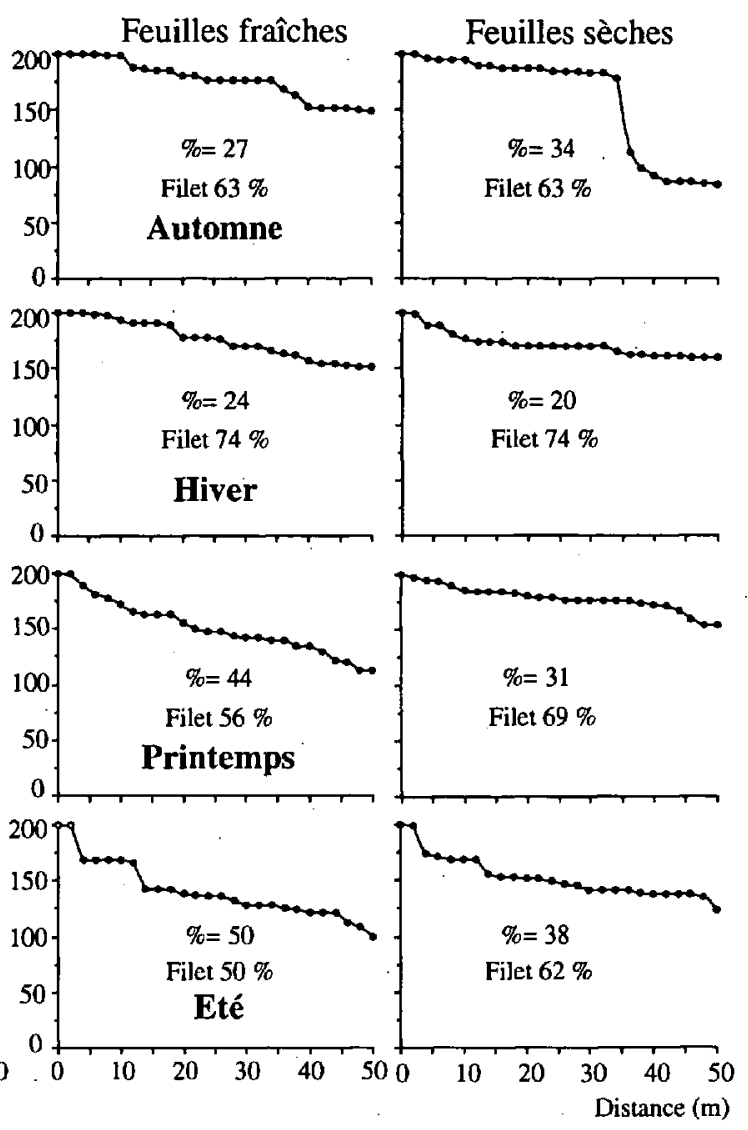

Fig. 2. Nombre de feuilles en dérive comme dans la figure 1, pour le secteur de $50 \mathrm{~m}$ du Bas Zeghzel. $\%_{0}=$ celui des feuilles récupérées dans le secteur Filet $=$ pourcentage des feuilles prises dans le filet à l'aval du secteur.

Fig. 2. Number of marked leaves transported as in Fig. 1 in the 50-m reach of the Lower Zeghzel. $\%=$ percentage of leaves recovered in the reach.

Filet $=$ percentage of leaves caught in the downstream net.

dont l'efficacité devient souvent plus importante au printemps et en été. L'efficacité du substrat minéral, constitué essentiellement de blocs, de galets et de gravier, reste assez faible par rapport aux autres structures et ne montre pas de relation particulière avec les variations saisonnières du débit : tantôt cette efficacité augmente, tantôt elle diminue. Les points stagnants ont également une efficacité de rétention assez faible, particulièrement dans le secteur amont où ils étaient peu abondants et relarguaient les feuilles avant $48 \mathrm{~h}$. Notons également, durant la période hivernale, le rôle joué par les algues filamenteuses dans la rétention des feuilles dans le Bas Zeghzel.
L'examen du tableau 2 montre que, dans la Branche Sud-Ouest, la rétention des feuilles de saule est plus importante que celle des feuilles de laurier en automne et en hiver. Au printemps et en été, lorsque le débit diminue, les feuilles fraîches des deux espèces sont plus retenues que les feuilles sèches.

Dans le bas Zeghzel, la comparaison des coefficients de rétention des deux essences végétales paraît plus difficile et la rétention des feuilles ne semble répondre à aucune règle générale.

Sur le plan trophique, on note que, durant la saison automnale, les dilacérateurs sont davantage 


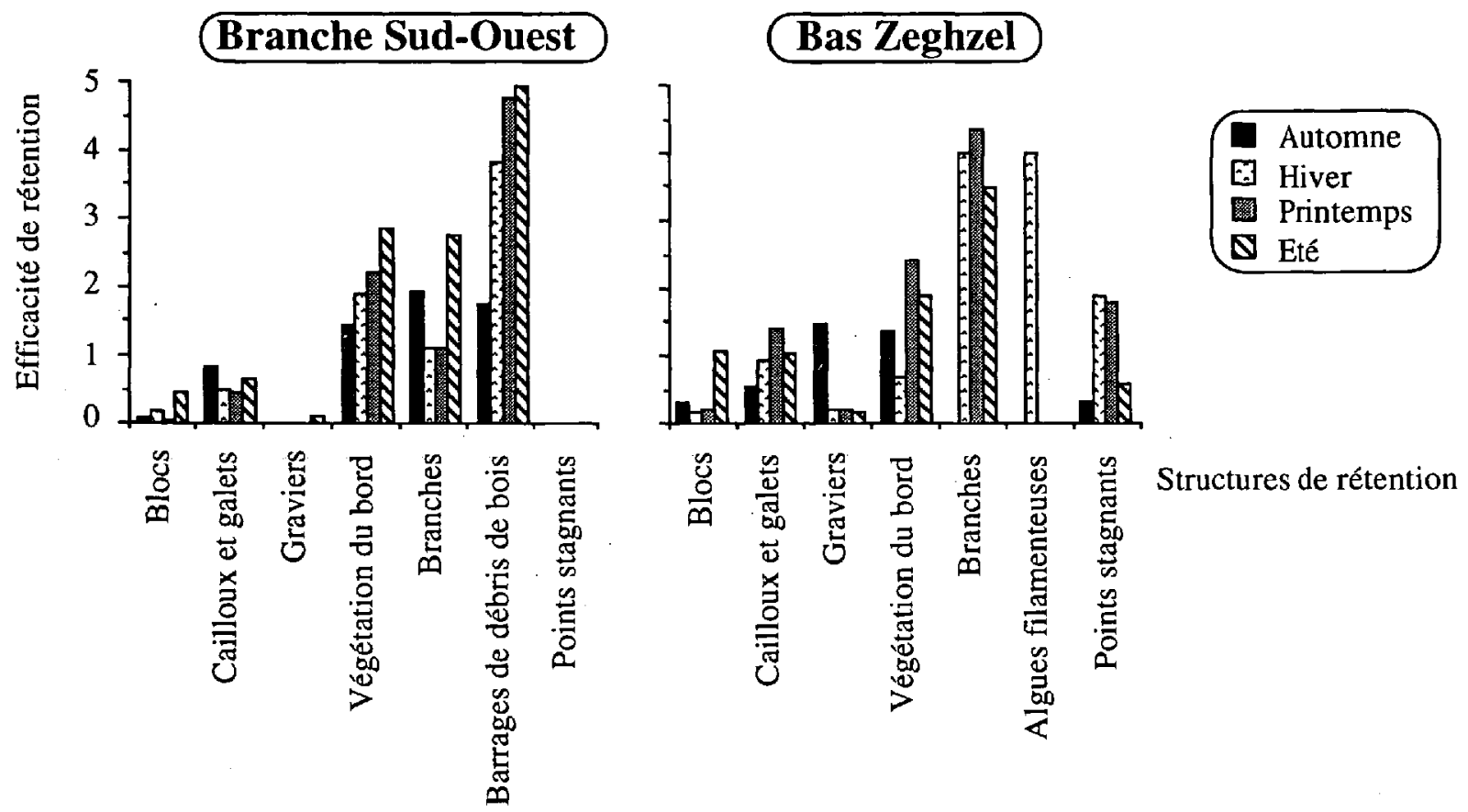

Fig. 3. Efficacité de rétention (définition dans le texte) des diverses structures dans les deux secteurs lors des quatre campagnes d'étude. Les cases vides indiquent une efficacité nulle.

Fig. 3. Retention efficiencies (definition in the text) of different features in the two reaches during the four sampling periods. The absence of a column indicates that no leaves were trapped.

représentés dans le secteur amont, particulièrement au niveau des structures de rétention qui se sont avérées les plus efficaces, telles les petits barrages de débris de bois et les branchages. Durant les autres saisons, la distribution des dilacérateurs suit approximativement le schéma précédent : dans la Branche Sud-Ouest, leur nombre et leur biomasse sont faibles au niveau des structures de rétention les moins efficaces et plus importants là où les structures retiennent le plus de feuilles. Dans le Bas Zeghzel, la rareté des dilacérateurs ne permet pas de dégager une telle relation et leur distribution s'avère aléatoire.

\section{Discussion}

Plusieurs auteurs ont également constaté une relation inverse entre débit et rétention des particules grossières de matière organique par le système aquatique (Cruz \& Post 1977, Malmqvist et al. 1978, Young et al. 1978, Prochazka et al. 1991, Petersen \& Petersen 1991). Prochazka et al (1991) ont constaté une nette différence dans le pouvoir rétentif de deux ruisseaux en Afrique du Sud, l'un avec un débit de $0,49 \mathrm{~m}^{3} \mathrm{~s}^{-1}$, l'autre $0,05 \mathrm{~m}^{2} \mathrm{~s}^{-1}$ et ils attribuent cette différence en grande partie au débit. Speaker et al. (1988) ont noté, aux USA, que Lookout 1 et 2 avec un débit de $0,14 \mathrm{~m}^{3} \mathrm{~s}^{-1}$, retiennent respectivement 367 et 394 feuilles, alors que Mack 1 et 2 , avec un débit de $0,034 \mathrm{~m}^{3} \mathrm{~s}^{-1}$, retiennent respectivement 1334 et 1433 feuilles sur des distances identiques. D'autres travaux insistent sur la largeur des cours d'eau. Selon Speaker et al. (1984), l'influence de la plupart des mécanismes intervenant dans le processus de rétention - existence de petits barrages de débris de bois, fréquence des obstacles, probabilité pour les particules de rencontrer ces obstacles... - tend à diminuer lorsque la largeur du cours d'eau augmente. Minshall et al. (1983) trouvent que, dans les montagnes de l'Oregon, la rétention diminue exponentiellement avec la largeur des cours d'eau. L'examen des travaux de Swanson et al. (1982) permet également d'y retrouver l'influence de la dimension du cours d'eau sur la rétention. 
Dans le Haut Zeghzel, quelle que soit la saison, le pourcentage des feuilles retrouvées et donc retenues dans le tronçon considéré est voisin de $100 \%$. Dans ce secteur, où la végétation riveraine est très dense et la largeur du cours d'eau faible, l'apport massif des débris organiques principalement issus de la végétation riveraine favorise l'installation des structures de rétention les plus efficaces, formées de débris de bois, et dont le rôle majeur a été largement mentionné dans la littérature (Bilby 1981, Bilby \& Likens 1980, Speaker et al. 1984, Winkler 1991). La répartition inégale de ces débris, liée au support minéral, explique les irrégularités des tracés de la fig. 1 : le tronçon commence sur 2 mètres par un secteur relativement dégagé, comprenant graviers et galets submergés avec quelques blocs arrondis ; suivent une zone plus hétérogène vers $4 \mathrm{~m}$, où se constituent ces barrages de débris ligneux, puis une nouvelle zone dégagée à $6 \mathrm{~m}$, plus étendue en hiver qu'au printemps. Se retrouvant à trois saisons successives, ces zones sont liées aux aspérités minérales stables du fond. En été, la réduction de la profondeur fait émerger d'autres aspérités dans les zones d'eau précédemment libre.

Dans le Bas Zeghzel, la pauvreté du couvert végétal couplée à un débit et à un rayon hydraulique plus importants entraîne l'absence de petits barrages de bois, ce qui affecte profondément la rétention des feuilles.

L'effet du débit s'observe aussi en comparant les saisons. En effet, lorsque le débit augmente, le rayon hydraulique augmente aussi et, par suite, la probabilité qu'a une particule d'atteindre le fond ou de rencontrer un obstacle diminue. Il est donc logique que la rétention des feuilles diminue en hiver et augmente en été (Cruz \& Post 1977, Young et al. 1978, Rounick \& Winterbourn 1983, King et al. 1987, Snaddon et al. 1992).

Snaddon et al. (1992) ont examiné l'influence des variations saisonnières du débit dans deux ruisseaux d'Afrique du Sud : à faible débit, la rétention des feuilles suivait un modèle exponentiel négatif, alors que, par débit plus important, la distribution des feuilles était aléatoire. Une tendance semblable se retrouve dans le Zeghzel où, bien que le modèle exponentiel négatif s'applique dans tous les cas, il est le meilleur en amont et par basses eaux en aval. Par débit plus important en aval, la rétention est linéaire, c'est-à-dire aléatoire. Le printemps en amont fait exception à cette règle, sans que nous ayons d'explication à proposer.

Dans le détail, les variations saisonnières du débit semblent influencer l'efficacité de rétention de certaines structures. Durant l'automne et l'hiver (périodes à débit important), l'efficacité de rétention de la végétation riveraine, des branchages et des débris de bois est plus faible que celle mesurée durant le printemps et l'été, périodes de faibles débits. L'augmentation du débit provoque une élévation du niveau de l'eau et, par suite, une submersion totale de certaines de ces structures. A l'inverse, une diminution du débit et par conséquent du niveau, provoque une émersion des structures ; dans ce cas, les feuilles flottant près de la surface auront donc plus de chances d'être capturées, comme nous l'avons remarqué plus haut. Snaddon et al. (1992) ont aussi constaté un changement dans l'efficacité de rétention de certaines structures en fonction du débit, mais il s'agissait de l'efficacité des bassins stagnants (structure de rétention la plus efficace dans leur étude), qui augmentait avec le débit. Aucune relation semblable n'apparaît dans le Zeghzel.

Quant à la nature et à la texture des feuilles utilisées, les légères différences de rétention peuvent peut-être s'expliquer par la plus grande flexibilité des feuilles de saule d'une part (hautes eaux), des feuilles fraîches d'autre part (basses eaux). Les feuilles flexibles s'entrelacent plus aisément autour des débris de bois et des branchages, ainsi que l'ont signalé Prochazka et al. (1991) en comparant les feuilles flexibles de Cunonia capensis L. et celles plus rigides de Brabejum stellatifolium L..

L'examen du nombre et de la biomasse des dilacérateurs associés à chacune des structures de rétention (Fig. 4) montre que ces organismes ne sont pas distribués de façon aléatoire mais colonisent la structure qui retient le plus de feuilles et l'habitat le plus approvisionné en matière végétale. Cette relation dilacérateurs - rétention des particules grossières avait été notée par d'autres auteurs (Minshall et al. 1983, Cummins et al. 1989, Prochazka et al. 1991, Hildrew et al. 1991). Quelle que soit la période d'étude, les dilacérateurs montrent une nette dominance dans le secteur amont du Zeghzel. Il apparaît donc que les fluctuations saisonnières du débit n'y affectent pas la distribution de ce groupe trophique. Dans le Bas Zeghzel, la densité et la biomasse de ce groupe restent toujours faibles, même 

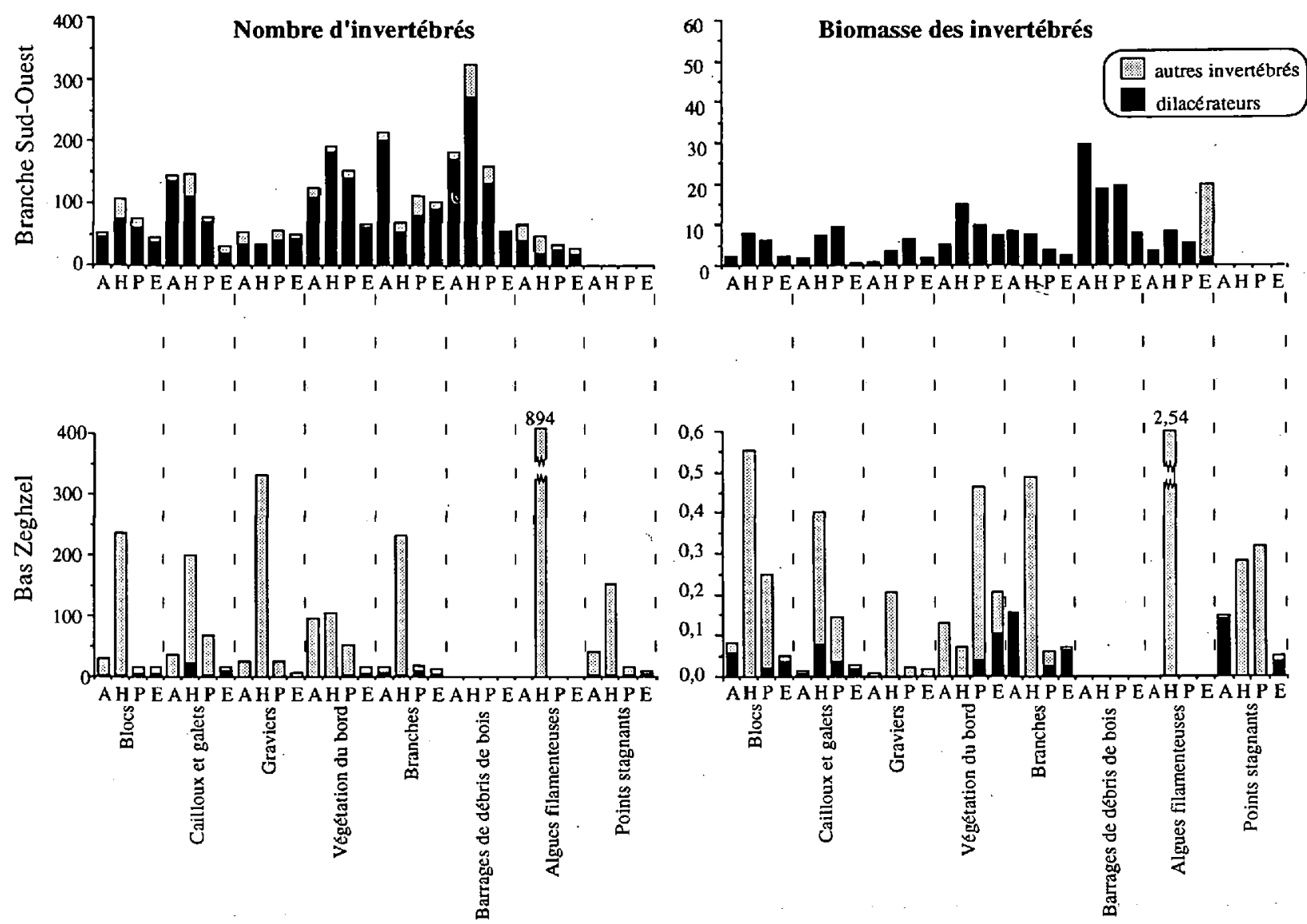

Fig. 4. Nombre et biomasse ( $\mathrm{g}$ de poids sec) des invertébrés collectés dans $0,1 \mathrm{~m}^{2}$ au niveau de chaque type de structure de rétention des deux secteurs lors des quatre périodes d'étude ( $\mathrm{A}$ : en automne, $\mathrm{H}:$ en hiver, $\mathrm{P}:$ au printemps, $\mathrm{E}:$ en été). Les cases vides indiquent l'absence de la structure dans le secteur concerné.

Fig. 4. Invertebrate numbers and biomass ( $\mathrm{g}$ dry weight) collected in $0.1 \mathrm{~m}^{2}$ of each type of retentive structure in the two reaches during the four sampling periods (A : autumn, $\mathrm{H}:$ winter, $\mathrm{P}:$ spring, $\mathrm{E}:$ summer). The absence of a column indicates the absence of the retentive structure in the reach considered.

durant le printemps et l'été lorsque le pouvoir rétentif de ce secteur s'améliore. Ceci renforce l'idée avancée par Chergui et al. (1993), qui expliquent le manque de dilacérateurs non pas tellement par une absence de substrats convenables, mais surtout par un manque de matière organique à exploiter.

\section{Conclusions}

L'influence du débit se manifeste de plusieurs façons différentes :

Le pouvoir de rétention de la zone amont à fajble débit et végétation surplombante est toujours supérieur à celui de la zone aval à plus fort débit et végétation espacée.

Au niveau des variations saisonnières, la baisse estivale du débit :

- diminue les distances parcourues par les feuilles,

- augmente la proportion de feuilles retenues dans le tronçon aval (elles sont toutes arrêtées à l'amont),

- accroît l'efficacité des structures de rétention les plus performantes et en fait émerger d'autres. 
Les feuilles flexibles ont tendance à être davantage retenues que les feuilles plus rigides.

La densité et la biomasse des macro-invertébrés dilacérateurs sont liées à l'efficacité de chaque structure habitée mais ne suivent pas les variations saisonnières du débit.

\section{Remerciements}

Les auteurs sont reconnaissants à un expert anonyme qui a attiré leur attention sur les conditions d'application du modèle exponentiel négatif de rétention des feuilles.

\section{Travaux cités}

Bilby R.E. 1981. - Role of organic debris dams in regulating the export of dissolved and particulate matter from a forested watershed. Ecology, 62 : 1234-1243.

Bilby R.E. \& Likens G.E. 1980. - Importance of organic debris dams in the structure and function of stream ecosystems. Eco$\log y, 61: 1107-1113$.

Boulton A.J. \& Boon P.I. 1991. - A review of methodology used to measure leaf litter decomposition in lotic environments : time to turn over an old leaf ? Aust. J. Mar. Freshwat. Res., $41: 1-43$.

Chergui H., Maamri A. \& Pattee E. 1993. - Leaf litter retention in two reaches of a Moroccan mountain stream. Limnologica, $23: 29-37$.

Chergui H. \& Pattee E. 1988. - The impact of benthic invertebrates on the breakdown of poplar leaves in the network of a large European river. Arch. Hydrobiol., 113 : 15-25.

Chergui H. \& Pattee E. 1991. - Dégradation des feuilles mortes allochtones dans le réseau de la Basse Moulouya, au Maroc. Acta oecol., 12 : 543-560.

Cummins K.W. \& Wilzbach A.M. 1985. - Field procedures for analysis of functional feeding groups of stream invertebrates. Appalachian Environmental Laboratory, University of Maryland, Frostburg. Cont. $\mathrm{N}^{\circ} 1611: 18 \mathrm{p}$.

Cummins K.W., Wilzbach M.A., Gates D.M., Perry J. \& Talliaferro W.B. 1989. - Shredders and riparian vegetation. BioScience, 39 (1) : 24-30.

Cruz A.A. de la \& Post H.A. 1977. - Production and transport of organic matter in a woodland stream. Arch. Hydrobiol., $80: 227-238$.

Fisher S.G. \& Likens G.E. 1973. - Energy flow in Bear Brook, New Hampshire : an integrative approch to stream ecosystem metabolism. Ecol. Monogr. $43:$ 421-439.

Hildrew A.G., Dobson M.K., Groom A., Ibbotson A., Lancaster J. \& Rundle S.D. 1991. - Flow and retention in the ecology of stream invertebrates. Verh. Internat. Verein. Limnol. 24 : 1742-1747.

King J.M., Day J.A., Davies B.R. \& Henshall-Howard M.P. 1987. - Particulate organic matter in a mountain stream in the south-western Cape. South Africa. Hydrobiologia, 154 : 165-187.

Merritt R.W. \& Cummins K.W. (eds) 1978. - An introduction to the aquatic insects of North America. Kendall/Hunth, lowa, USA : $441 \mathrm{p}$.
Malmqvist B., Nilsson L.M. \& Svensson B.S. 1978. - Dynamics of detritus in a small stream in southern Sweden and its influence on the distribution of the bottom animal communities. Oikos, $31:$ 3-16.

Minshall G.W., Petersen R.C., Cummins K.W., Bott T.L., Sedell J.R., Cushing C.E. \& Vannote R.L. 1983. - Interbiome comparison of stream ecosystem dynamics. Ecol. Monogr., 53 : $1-25$.

Oertli B. 1991, - Oak leaf litter processing and associated macroinvertebrates in two ponds near Geneva (Switzerland). Verh. Internat. Verein. Limnol., 24 : 1543-1546.

Pattee E., Bornard C. \& Mourelatos S. 1986. - La décomposition des feuilles mortes dans le réseau fluvial du Rhône : influence du milieu et principaux agents responsables. Revue fr. Sci. Eau, $5:$ 45-74.

Petersen L.B.M. \& Petersen R.C. 1991. - Short term retention properties of channelized and natural streams. Verh. Internat. Verein. Limnol., 24 : 1756-1759.

Post A.H. \& Cruz A.A de la, 1977. - Litter fall, litter decomposition and flux of particulate organic material in a coastal plain stream. Hydrobiologia, 55 : 201-207.

Prochazka K., Stewart B.A. \& Davies B.R. 1991. - Leaf litter retention and its implication for shredder distribution in two headwater streams. Arch. Hydrobiol., $120: 315-325$.

Rounick J.S. \& Winterbourn M.J. 1983. - Leaf processing in two contrasting beech forest streams : effects of physical and biotic factors on litter breakdown. Arch. Hydrobiol., 96 : 448-474.

Snaddon C.D., Stewart B.A. \& Davies B.R. 1992. - The effect of discharge on leaf retention in two headwater streams. Arch. Hydrobiol., 125 : 109-120.

Speaker R.W., Moore K. \& Gregory S. 1984. - Analysis of the process of retention of organic matter in stream ecosystems. Verh. Internat. Verein. Limnol., 22 : 1835-1841.

Speaker R.W., Luchessa K.J., Franklin J.F. \& Gregory S.V. 1988. - The use of plastic strips to measure leaf retention by riparian vegetation in a coastal Oregon stream. Amer. Midl. Nat., $120: 22-31$.

Swanson F.J., Gregory S.V., Sedell J.R. \& Campbell A.G. 1982. - Land-water interactions : the riparian zone. In : Edmonds R.L. (ed) Analysis of coniferous forest ecosystems in the Western United States. US/IBP Synthesis Series, $14: 267-291$.

Tachet H., Bournaud M. \& Richoux P. 1980. - Introduction à l'étude des macroinvertébrés des eaux courantes. Assoc. Fr. Limnol. et Univ. Lyon : $156 \mathrm{p}$.

Webster J.R. \& Benfield F., 1986. - Vascular plant breakdown in freshwater ecosystems. Ann. Rev. Ecol. Syst., $17: 567-594$.

Winkler G. 1991. - Debris dams and retention in a low order stream (a backwater of Oberer Seebach - Ritrodat-Lunz Study Area, Austria). Verh. Internat. Verein. Limnol., 24 : 1917-1920.

Winterbourn M.J., Rounick J.S. \& Cowie B. 1981. - Are New Zeland stream ecosystems really different? N.Z.J. Mar. Freshwat. Res., $15:$ 321-328.

Young S.A. Kovalak W.P. \& Del Signore K.A. 1978. - Distance travelled by autumn-shed leaves introduced into a woodland stream. Am. Midl. Nat., $100: 217-222$. 